\title{
KONGRESSKALENDER
}

\section{Mai 2015}

15.05.2015, Dresden

20. Dresdner Frühjahrstagung für Forensische Psychiatrie

Methamphetamin („Crystal“) - kriminologische, juristische und psychiatrische Aspekte

Dr. Lau, Dr. Lammel, Dr. Sutarski, Prof. Dr. Bauer

Information: www.forensik-dresden.de

20.-22.05.2015, Zürich, Schweiz

7. Internationales Symposium Forensische Psychiatrie

World Trade Center

Prof. Endrass, Dr. Rossegger, Prof. Urbaniok

Information: www.forensiktagung.ch

\section{Juni 2015}

08.-10.06.2014, Stockholm, Schweden

The Stockholm Criminology Symposium

Opportunities for crime and situational crime prevention.

City Conference Center, Stockholm

Information: www.criminologysymposium.com

12.06.2015, Berlin-Dahlem

19. Berliner Junitagung für Forensische Psychiatrie und Psychologie

Mord und Totschlag -

Psychologische und juristische Erfassung Institut für Forensische Psychiatrie der Charité

Information: www.forensik-berlin.de (Veranstaltungen)

16.06.-18.06.2015, Manchester, UK

15th Annual IAFMHS Conference

Forensic Mental Health Services

University of Manchester

Information: www.iafmhs.org

\section{Juli 2015}

\section{2.-17.07.2015, Wien, Österreich}

34th Congress of the IALMH

International Academy of Law and Mental Health

Hotel Intercontinental

Information: www.ialmh.org

\section{August 2015}

04.-07.08.2015, Nürnberg

European Association of Psychology and

Law (EAPL)

Annual Conference

Prof. F. Lösel, Dr. D. Bender

Information: www.eapl.eu

\section{September 2015}

02.-05.09.2015, Porto, Portugal

European Association of Criminology

15th Annual ESC Conference: Criminology as unitas multiplex:

theoretical, epistemological and methodological developments

University of Porto

Information: http://www.esc-eurocrim.org/

\section{4.-26.09.2015, Köln}

Kriminologische Gesellschaft (KrimG)

14. Wissenschaftliche Fachtagung

„Krise - Kriminalität - Kriminologie“

Prof. Dr. F. Neubacher

Information: http://www.kriminologie.uni-koeln.de/krimg-tagung2015.html

\section{Oktober 2015}

08.-10.10.2015, München

30. Herbsttagung für Forensische Psychiatrie

Prof. N.N.

Abteilung für Forensische Psychiatrie der Universität München

Information: www.forensik-muenchen.de

14.-17.10.2015, Montréal, Canada

Association for the Treatment of Sexual Abusers (ATSA)

34th Annual Research and Treatment Conference

Le Centre Sheraton Montreal Hotel

Information: www.atsa.com/conference

22.-25.10.2015, Fort Lauderdale, Florida (USA)

American Academy of Psychiatry and the Law

46th Annual Meeting (with APA)

Marriott Harbor Beach Resort

Information: www.AAPL.org

\section{November 2015}

12.-13.11.2015, Göttingen, Klinik für Forensische Psychiatrie

Tagung Empirische Forschung in der Forensischen Psychiatrie, Psychologie und Psychotherapie

Prof. Dr. J.L. Müller

Information: http://forensik-umg.bplaced.net/

18.-21.11.2015 Washington D.C. (USA)

American Society of Criminology - Annual Meeting

Neglected Crimes, Groups, Causes and Policy Approaches

Information: http://www.asc41.com/annualmeeting.htm

Ein internationaler kriminologischer Kongresskalender findet sich unter http://www.asc41.com/caw.html

Hinweise für den Kongresskalender bitte an: hans-ludwig.kroeber@charite.de 